\title{
ESTRATEGIAS DE MERCADO PARA EL FRIJOL CENTROAMERICANO'
}

\author{
Lourdes Martínez ${ }^{2}$, Richard Bernsten ${ }^{2}$, Miguel Zamora ${ }^{2}$
}

\section{RESUMEN}

Estrategias de mercado para el frijol centroamericano. En los últimos años, el sub-sector de frijol en Centro América ha sido testigo de varios cambios dinámicos. Si no se encuentran medidas para incrementar la competitividad regional de este sub-sector, los países centroamericanos experimentarán impactos negativos en las áreas sociales y económicas de considerable magnitud, especialmente ahora que Centro América necesita ajustar su economía a los nuevos requerimientos de mercados mas abiertos, como el Tratado Centroamericano de Libre Comercio con USA (TLC). Vendedores de frijoles a nivel intermediario y de consumo final, así como técnicos de oficinas gubernamentales dedicadas al mercadeo de frijoles en Centro América fueron entrevistados para documentar los cambios experimentados, identificar los obstáculos que frenan la viabilidad del sub-sector e identificar las oportunidades de incremento de mercados en el futuro para los productores y vendedores de frijol. Los resultados muestran que para que continúen siendo competitivos, los países centroamericanos deben incrementar la productividad a través del apoyo a las investigaciones para el mejoramiento de variedades de frijol, especialmente aquellas que los consumidores prefieren por características específicas (e.g. color). También es imprescindible el fortalecimiento de los programas de distribución de variedades mejoradas a los productores; las mejoras en la transmisión de información de mercado a los productores; el fortalecimiento de los contactos entre productores, comercializadores a nivel mayorista y supermercados; y una política más específica de apertura de mercados de exportación, especialmente con los Estados Unidos.

\begin{abstract}
Market strategies for Central American dry beans. In the past few years, the dry bean sub-sector in Central America has witnessed many dynamic changes. Unless we find ways to increase the competitiveness of the regional bean sub-sector, Central American countries will likely experience significant negative social and economic impacts, especially since these countries are facing the challenge of adjusting to new open markets, such as the Central American Free Trade Agreement (CAFTA). Bean traders, retailers, and knowledgeable government official in Costa Rica, El Salvador, Honduras, Guatemala, and Nicaragua were interviewed to document these changes, identify constraints that threaten the viability of the sub-sector, and assess future opportunities for increasing bean market shares. The results indicate that to remain competitive in the future, Central American countries must increase productivity by continuing to support dry bean research (i.e., develop improved varieties with characteristics that consumers prefer such as grain color); strengthen programs for distributing improved varieties to producers; provide producers with better market information; develop stronger linkages between producers, packers, wholesalers, and supermarkets; and aggressively target the U.S. export market.
\end{abstract}

Key words: subsector analysis, strategic planning, dry bean, Central America.

Palabras claves: análisis de subsector, planeamiento estratégico, frijoles, Centro América.

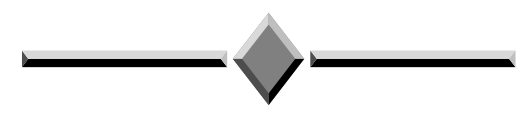

1 Recibido para publicación el 22 de junio del 2004. Trabajo presentado en la L Reunión Anual del PCCMCA. San Salvador, El Salvador. Esta publicación fue posible gracias al soporte proveído al Bean/Cowpea Collaborative Research Support Program (CRSP) por la Oficina de Crecimiento Económico, Agricultura y Comercio, la Agencia Americana para Ayuda Internacional (USAID), bajo los términos del contrato No. GDG-G-00-02-00012-00. Las opiniones expresadas en el presente trabajo son de los autores y no representan necesariamente los puntos de vista de la USAID.

2 Departamento de Economía Agrícola, Michigan State University, East Lansing, MI 48824 (con la colaboración de J.C. Rosas, Escuela Agrícola Panamericana, Zamorano, Honduras, en la edición del documento).Correo electrónico: bernsten@msu.edu, miguelzamora@yahoo.com. 


\section{INTRODUCCIÓN}

El sub-sector frijolero es estratégicamente importante para los países centroamericanos. Los frijoles son un componente básico en la dieta centroamericana, una importante fuente de proteína vegetal para la población, especialmente de escasos recursos en áreas urbanas y rurales, y finalmente, una fuente importante de ingresos para los pequeños productores.

A pesar de la importancia de los frijoles en Centro América, desde el inicio de los años noventas, el subsector de frijol ha sido blanco de reformas que incluyeron la gradual eliminación de las agencias de mercadeo estatales, la disminución del apoyo gubernamental a la investigación y extensión agrícola tradicional, y un progresivo movimiento hacia la firma de tratados de libre comercio en la región y fuera de la misma (Martel et al. 2000). Sin embargo, si bien los gobiernos en Costa Rica, El Salvador, Guatemala, Honduras y Nicaragua han aplicado estas medidas políticas, las importaciones de frijoles se incrementaron constantemente durante los años 1990 y los productores de frijol aún enfrentan dificultades para competir con el mercado internacional.

La baja productividad ${ }^{1}$ es el mayor obstáculo centroamericano para obtener una producción suficiente para satisfacer los requerimientos de los mercados locales. Recientemente, los bajos rendimientos relativos y los frecuentes desastres naturales (huracanes, inundaciones, sequías) han provocado que a estos países les sea imposible satisfacer sus demandas locales, lo cual ha requerido que se importen frijoles para satisfacer la demanda de sus consumidores.

La baja productividad también limita la habilidad de los productores centroamericanos para competir en el mercado internacional. Esta situación ha motivado una creciente preocupación que la integración global de los mercados provocará una creciente disponibilidad de frijoles con mejores calidades y a precios mas bajos, lo que a su vez implicara el reemplazo de la producción local y el futuro empobrecimiento de las áreas rurales.

Si bien algunos investigadores argumentan que la reciente expansión de las actividades de procesamiento podrían incrementar la demanda de frijoles locales, los productores de estos países podrían no estar beneficiándose de este crecimiento. Por ejemplo, en un estudio realizado en Guatemala, se encontró que las empresas productoras de enlatados utilizaban muy poco frijol

\footnotetext{
1 En el periodo 1999-2001, el rendimiento promedio en Costa Rica fue $583 \mathrm{~kg} / \mathrm{ha}$, en El Salvador $877 \mathrm{~kg} / \mathrm{ha}$, en Guatemala $689 \mathrm{~kg} / \mathrm{ha}$, en Honduras $688 \mathrm{~kg} / \mathrm{ha}$ y en Nicaragua $746 \mathrm{~kg} / \mathrm{ha}$.
}

producido localmente, debido a los altos costos del frijol en Guatemala, comparado con los bajos precios de productos importados y el bajo costo de procesar frijoles partidos provenientes de los Estados Unidos (Estrada-Valle 2001). En consecuencia, el crecimiento de la demanda de frijoles por parte de las empresas procesadoras dependerá de la disponibilidad de frijoles de bajos precios que también satisfagan los requerimientos de calidad demandados por los consumidores.

Actualmente existe muy poca información sistematizada, actualizada, e integrada acerca del sub-sector frijolero en la región, especialmente relacionada a problemas de mercadeo (incluyendo oportunidades de comercio en la región y las posibilidades de la región para exportar a otros países fuera de la región centroamericana). Para cubrir éste vacío de información, el presente trabajo presenta: 1) una descripción breve de la industria de productos procesados en la región, 2) un análisis de las tendencias de importación y precios en la región, y 3) una propuesta de estrategias para ayudar a incrementar la competitividad de la región.

\section{METODOLOGÍA}

El presente trabajo utiliza la metodología conocida como reconocimiento rápido para documentar el status del sub-sector del frijol en Centro América. La metodología de reconocimiento rápido permite establecer "la búsqueda de oportunidades para el mejoramiento del funcionamiento del sistema, así como alternativas para explorar alternativas que no están siendo explotadas o bien no han sido consideradas aun para acceder a nuevos mercados, generar ofertas significativamente más elevadas, y mejorar la eficiencia del sistema alimenticio" (Holtzman 1986).

El área geográfica para la conducción del reconocimiento rápido incluyó cinco capitales de países de Centro América. Las capitales de estos países fueron seleccionadas porque una proporción grande de la producción es vendida en estas ciudades, y también porque en las ciudades capitales están situadas las principales oficinas de gobierno, y los principales procesadores, supermercados, importadores y exportadores. Los entrevistados fueron seleccionados entre miembros de los siguientes grupos: 1) vendedores mayoristas en las ciudades capitales (importadores, exportadores, vendedores en mercados centrales), 2) empacadores, 3) procesadores, 4) administradores de supermercados, 5) agentes de gobierno, y 6) mejoradores de variedades de frijol. El objetivo del recononocimiento rápido fue utilizar información secundaria e información obtenida de las entrevistas a conocedores del mercado de frijol para describir 
la estructura del sub-sector del frijol en Centro América, identificar obstáculos para incrementar la competitividad del sector, y finalmente, identificar puntos críticos de intervención para superar las barreras.

Un total de 57 entrevistas fueron conducidas en Centro América (Cuadro 1). Las entrevistas fueron personales y se llevaron a cabo durante el mes de julio del 2002. El proceso de entrevistas que tomó una semana en cada país, comenzó en Costa Rica, seguido de Nicaragua, El Salvador, Guatemala y Honduras.

\section{RESULTADOS Y DISCUSIÓN}

\section{Situación de la industria del frijol en Centro América}

Los empacadores y los procesadores de frijoles representan la industria de valor agregado en Centro América.

\section{Empacadores}

En áreas rurales, los empacadores de frijoles compran frijoles de intermediarios y los limpian, clasifican, empacan y redistribuyen a supermercados, vendedores de mercados, pequeñas tiendas (pulperías), y algunas veces a hoteles y restaurantes. En mayor medida, los empacadores de frijoles se encuentran embolsando para cadenas de supermercados que también se encuentran promocionando sus marcas privadas.
Según lo expresado por los administradores de empresas empacadoras entrevistados, la creciente consolidación de los supermercados les ha afectado en diferentes medidas. Primero, los supermercados se han convertido en importantes compradores en el sistema alimenticio. Esta característica les da poder de decidir los términos de pago (en algunos casos los supermercados se demoran entre 30 a 45 días para pagar a los empacadores). Segundo, como no existe un contrato con los supermercados para la venta del producto, si el supermercado no vende el producto, el supermercado tiene el derecho de remover el producto de sus estantes y cargar el costo al empacador. Tercero, el costo para entrar a vender a los supermercados es muy alto. Los supermercados requieren a los vendedores el pago para asegurar un espacio en los estantes, esperan que los empacadores realicen promociones, y generalmente el tiempo de pago se extiende más de lo acordado.

Durante las entrevistas se pudo constatar que algunos de los empacadores entrevistados no estaban vendiendo sus productos en los supermercados, y la única alternativa era la venta a pequeños negocios (pulperías), a hoteles y restaurantes o en ciudades secundarias a la ciudad capital. Por otro lado, también existen grandes beneficios cuando se puede vender a los supermercados. El volumen de venta es grande y la posibilidad de rotación de dinero es mas rápida (el producto se mueve mucho mas rápido cuando es ofrecido en los supermercados). Además, las compañías que empacan marcas privadas para los supermercados generalmente venden un volumen más grande, por el cual reciben una comisión y ganan acceso al espacio en el supermercado.

Cuadro 1. Entrevistas por grupo de actores efectuada en Centromérica para documentar el status del sector frijolero. Centroamérica, 2002.

\begin{tabular}{lccccc}
\hline \multicolumn{1}{c}{ Entrevistas } & Costa Rica & Salvador & Guatemala & Honduras & Nicaragua \\
\hline $\begin{array}{l}\text { Mayoristas, importadores } \\
\text { y/o exportadores }\end{array}$ & 2 & 3 & 1 & 2 & 1 \\
$\begin{array}{l}\text { Empacadores de frijoles } \\
\text { Procesadores de frijoles }\end{array}$ & 5 & 2 & 1 & 4 & 1 \\
$\begin{array}{l}\text { Administradores de } \\
\text { Supermercados }\end{array}$ & 2 & 2 & 2 & 1 & 0 \\
$\begin{array}{l}\text { Agentes de gobierno } \\
\text { Mejoradores de frijoles e }\end{array}$ & 2 & 2 & 2 & 1 & 1 \\
investigadores* & 2 & 1 & 2 & 1 & 3 \\
Total & 15 & 1 & 3 & 3 & 2 \\
\hline
\end{tabular}

*Incluye a investigadores de mercado. 
Según lo recabado en la investigación, aproximadamente $30 \%$ de los productos empacados eran de marcas privadas de los supermercados, el resto $(70 \%)$ eran marcas de los empacadores.

Para que los frijoles locales sean competitivos con los frijoles importados, los empacadores de frijoles necesitan una oferta constante a lo largo del año. También, los requerimientos para satisfacer estándares privados sobre calidad de frijoles han ido incrementándose en los últimos años (color y tamaño).

\section{Procesadores}

En la región, aproximadamente 15 firmas procesan frijoles en diferentes productos (frijoles enteros enlatados, refritos y en polvo). De este número, 10 procesadores que representan a los más grandes y con mayor proporción del mercado se encuentran en Guatemala (7), Costa Rica (2) y Honduras (1). Las mayores procesadoras son multinacionales, que producen mayormente frijoles enlatados y generalmente venden sus productos a través de todos los supermercados en Centro América y algunas también exportan a los Estados Unidos (Cuadro 2).

Cuadro 2. Procesadores de frijol por país de origen y relación con empresas internacionales. Centoamérica, 2002a.

\begin{tabular}{|c|c|c|}
\hline Procesador & $\begin{array}{l}\text { Presentación } \\
\text { del producto }\end{array}$ & $\begin{array}{l}\text { Participación } \\
\text { internacional }\end{array}$ \\
\hline \multicolumn{3}{|l|}{ Costa Rica } \\
\hline Alimer & Enlatado & Firma local \\
\hline Conservas del Valle S.A. & Enlatado & $\begin{array}{l}\text { Unilever UK/ } \\
\text { Holanda }\end{array}$ \\
\hline $\begin{array}{l}\text { Industrias Sanso S.A. } \\
\text { El Salvador }\end{array}$ & Enlatado & Firma local \\
\hline Grupo Coscafé & Polvo & Firma local \\
\hline Garmol & Enlatado & Firma local \\
\hline Industrias LYA SA & Enlatado & Firma local \\
\hline \multicolumn{3}{|l|}{ Guatemala } \\
\hline Agroindustrias Lozano S.A. & Enlatado & Firma local \\
\hline Alisa de Guatemala & Enlatado & Firma local \\
\hline Alimentos Maravilla & Enlatado & Del Monte USA \\
\hline Empaques Agroindustriales & Enlatado & CARHCO \\
\hline Malher Sucesores y Cia. & Enlatado & Firma local \\
\hline Empacadora Toledo & Enlatado & Firma local \\
\hline Empresas Integradas & Enlatado & CARHCO \\
\hline $\begin{array}{l}\text { Alimentos Kern de } \\
\text { Guatemala }\end{array}$ & Enlatado & Ducal USA \\
\hline \multicolumn{3}{|l|}{ Honduras } \\
\hline Alimentos Natura & Empaque flex. & $\begin{array}{l}\text { Unilever UK/ } \\
\text { Holanda }\end{array}$ \\
\hline
\end{tabular}

a Esta lista representa productos que son producidos en América Central y que son vendidos en las mayores cadenas de supermercados en las ciudades capitales. Aunque no es una lista completa, la misma incluye los mayores productores locales de productos de valor agregado. No incluye productos importados.
La presencia de marcas privadas de los supermercados en productos enlatados ha afectado a la industria procesadora pero en menor medida, ya que los consumidores Centroamericanos tienen una marcada preferencia por ciertas marcas. El impacto de nuevos competidores ha sido menos crítico para aquellas empresas con marcas posicionadas en el mercado y con amplia aceptación (Naturas, Del Monte). Para mantener su participación en el mercado, estas marcas han introducido nuevos productos (frijoles con vegetales, frijoles con chorizo) que son muy aceptados por los consumidores en Centro América.

En contraste, la perspectiva de aumento de las ventas vía exportaciones es menos prometedora. Los procesadores entrevistados en Centro América dijeron que es difícil exportar a los Estados Unidos, Canadá y Europa porque en estos países existen una gran cantidad de marcas establecidas en el mercado; estos países también imponen altas barreras a la entrada para los productos de Centro América. Por otro lado, en ciertas ciudades como Nueva Jersey, el sur de California, Miami, y Chicago, donde existe una concentración grande de hispanos y la preferencia por los frijoles de Centro América es fuerte, se puede hablar de la existencia de un nicho de mercado para vender productos procesados de frijol (Batres-Marquez et al. 2001).

De acuerdo a lo expresado por los procesadores entrevistados, el mayor obstáculo para incrementar las ventas en el mercado centroamericano es la preferencia de los consumidores.

Los centroamericanos generalmente no compran productos enlatados y estos productos tienen un precio mucho mayor al de los productos empacados.

Sin embargo, cambios en aspectos demográficos (mayor número de mujeres en el mercado laboral que limita el tiempo para la preparación de alimentos), el incremento relativo de los salarios, y las economías de escala de las grandes empresas procesadoras (economías de escala que reducen los precios finales) ayudarán, a largo plazo, al incremento de la demanda de productos procesados. Los procesadores entrevistados también remarcaron que para satisfacer la demanda se necesita una oferta constante de frijoles a lo largo del año, así como precios con menos variaciones durante el año.

\section{Importaciones de frijoles en Centro América}

Recientemente, la región centroamericana ha incrementado su dependencia de importaciones de frijoles para satisfacer la demanda de sus consumidores. Durante el periodo 1994-1996 y 1999-2001, la tasa 
anual de crecimiento de las importaciones fue de $18 \%$, con un incremento total de $127 \%$ (Cuadro 3). En términos de volumen, la importación total de la región se incrementó de un promedio anual de 22.293 TM a 50.586 TM. Sin embargo, es de destacar que la tendencia de las importaciones varió grandemente de país a país durante este periodo. Por ejemplo, durante el periodo 1994 a 2001, las importaciones de frijoles en Guatemala se incrementaron a una tasa anual de $28 \%$, seguidos por Costa Rica (24\%), Nicaragua (16\%), El Salvador $(15 \%)$ y Honduras $(9 \%)$.

Cuadro 3. Tendencia de importaciones de frijol. Centroamérica, 1994-2001.

\begin{tabular}{|c|c|c|c|c|c|}
\hline \multirow[t]{2}{*}{ País } & \multicolumn{2}{|c|}{$\begin{array}{c}\text { Promedio } \\
\text { Anual a b }\end{array}$} & \multicolumn{2}{|c|}{$\begin{array}{c}\text { Cambio } \\
\text { Equivalente }^{c}\end{array}$} & \multirow[t]{2}{*}{ Oferta } \\
\hline & $\begin{array}{l}1994- \\
1996\end{array}$ & $\begin{array}{l}\text { 1999- } \\
2001\end{array}$ & Anual & Total & \\
\hline Costa Rica & 8.786 & 25.372 & $24 \%$ & $189 \%$ & $61,4 \%$ \\
\hline El Salvador & 7.284 & 14.928 & $15 \%$ & $105 \%$ & $18,8 \%$ \\
\hline Guatemala & $1, .28$ & 4.849 & $28 \%$ & $240 \%$ & $5,5 \%$ \\
\hline Honduras & 1.918 & 2.966 & $9 \%$ & $55 \%$ & $5,9 \%$ \\
\hline Nicaragua & 2.877 & 5.953 & $16 \%$ & $107 \%$ & $4,0 \%$ \\
\hline Centro América & 22.293 & 50.586 & $18 \%$ & $127 \%$ & $11,7 \%$ \\
\hline
\end{tabular}

a Toneladas métricas.

b La información que los países centroamericanos proveen al SIECA no incluye el comercio generado por actividades de empaque (ejemplo, importaciones de frijoles en sacos en vez de containers). En el periodo en estudio esta información no fue proporcionada por Guatemala, Honduras y Nicaragua.

c Importaciones como proporción total de la oferta de frijoles en el periodo 1999-2001.

Fuente: Sistema de Estadísticas de Centro América (SIECA 2003).

En el periodo 1999-2001, Costa Rica representó casi la mitad del total de las importaciones (48\%) de la región, seguido por El Salvador (27\%), Nicaragua (11\%), Guatemala (9\%), y Honduras (5\%) (Figura 1).

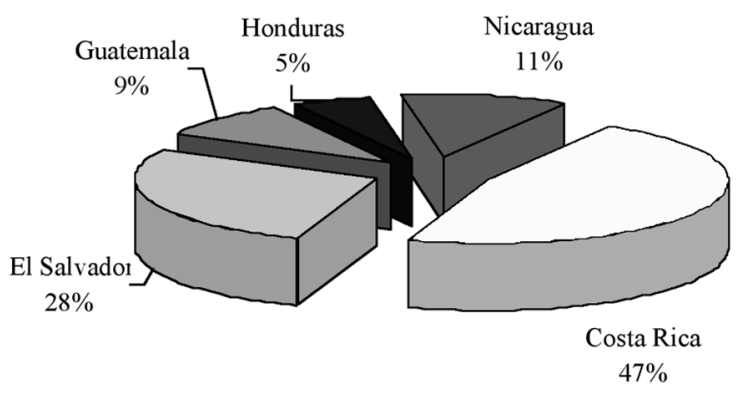

Figura 1. Porcentaje de importaciones anuales de frijol por país. Centro América, 1999-2001.

Fuente: Informaciones de SIECA (2003).
La fuente de provisión depende primeramente de la disponibilidad de la clase de frijol deseada y del precio. En el 2001, Costa Rica se abasteció de frijoles de Argentina (39\%), Nicaragua (22\%), Guatemala (19\%) y de EE.UU (13\%); El Salvador mayormente de Nicaragua $(62 \%)$ y Honduras (32\%); Guatemala se proveyó de EE.UU (59\%) y Canadá (39\%); Honduras en un 99\% compró de Nicaragua; y Nicaragua se proveyó en un $94 \%$ de los EE.UU (Cuadro 4).

Cuadro 4. Fuente de importaciones de frijoles por país de origen. Centroamérica, 2001 a.

\begin{tabular}{lccccc}
\hline $\begin{array}{c}\text { País de } \\
\text { origen }\end{array}$ & $\begin{array}{c}\text { Costa } \\
\text { Rica }\end{array}$ & $\begin{array}{c}\text { El } \\
\text { Salvador }\end{array}$ & Guatemala Honduras & Nicaragua \\
\hline Costa Rica & N/A & $1,9 \%$ & 0 & $<1 \%$ & 0 \\
Guatemala & $18,8 \%$ & $4,6 \%$ & N/A & 0 & 0 \\
Honduras & $1,5 \%$ & $31,5 \%$ & 0 & N/A & 0 \\
Nicaragua & $22,2 \%$ & $61,6 \%$ & 0 & $98,8 \%$ & N/A \\
Argentina & $38,8 \%$ & 0 & $2,3 \%$ & 0 & $2,6 \%$ \\
Canada & $5,9 \%$ & 0 & $38,8 \%$ & $<1 \%$ & $3,8 \%$ \\
Estados & \multicolumn{7}{c}{0} & & & \\
Unidos & $12,9 \%$ & $<1 \%$ & $58,9 \%$ & $<1 \%$ & $93,6 \%$ \\
\hline
\end{tabular}

a Porcentaje del total de importaciones en el año 2001. Fuente: SIECA (2003).

La mayoría de los países centroamericanos tienden a proveerse de frijoles en la región. Sin embargo, la disponibilidad de frijoles a menores costos en países como Argentina y los EE.UU, ha influenciado que Centro América se provea de estos países. Típicamente, el comercio con EE.UU, Argentina y Canadá comienza en abril, cuando el abastecimiento de la región alcanza sus niveles mas bajos y los precios alcanzan sus niveles más altos, y continua hasta aproximadamente el mes de diciembre ${ }^{2}$.

Para reducir la tendencia creciente a importar frijoles de países fuera de la región, los países centroamericanos han impuesto aranceles a la importación que promedian en un $30 \%$ en toda la región. Según los entrevistados, esta medida no ha logrado su objetivo de reducir las importaciones e incrementar la producción de la región. Según los importadores entrevistados, los frijoles se importan de fuera de la región cuando no existe oferta suficiente en Centro América, por lo tanto los aranceles afectan principalmente a los consumidores finales quienes tienen que pagar más por los frijoles.

\footnotetext{
2 El periodo de cosecha en Argentina es de abril a julio, mientras que en EEUU y Canadá es de setiembre a octubre.
} 


\section{Tendencia de los precios del frijol}

Durante el periodo 1997-2002, los precios mayoristas del frijol han variado por país y de acuerdo a la clase de frijol. Los precios de los frijoles negros en Guatemala han sido bien variables, alcanzando un máximo de $1,300 \mathrm{US} \$ / \mathrm{t}$ en enero de 1999 , cayendo a 500 US\$/t en mayo del 2000, nuevamente subiendo a 900 US\$/t en setiembre del 2001, y finalmente reduciéndose a 500 US $\$ / t$ en octubre del 2002. En contraste, mientras el precio del frijol negro en Costa Rica ha sido menos volátil, los precios se incrementaron desde 500 US\$/t en enero de 1998 a 1,000 US $\$ / t$ en mayo de 1999 , cayendo a 700 US\$/t en setiembre del 2000 , y finalmente incrementándose a 900 US\$/t en octubre del 2002 (Figura 2). Interesante, si bien Costa Rica importa una cantidad substancial de frijoles $(60,7 \%$ del total de oferta), los precios mayoristas han sido mayores que los otros países centroamericanos.

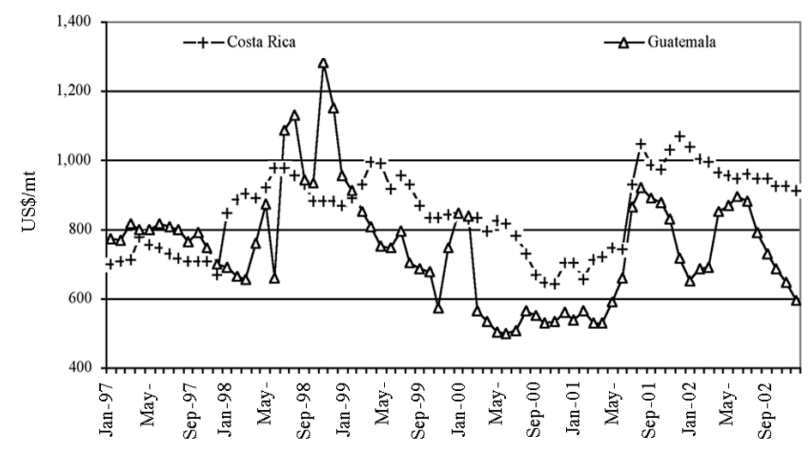

Figura 2. Precios nominales mayoristas de frijol negro. Costa Rica y Guatemala, 1997-2002.

Fuente: Datos de CORECA (2003).
En Honduras, Nicaragua y El Salvador, el precio del frijol rojo tuvo una variación importante en el periodo 1997-2002. Si bien los precios fueron muy volátiles, los precios mayoristas han tenido la tendencia a disminuir en el periodo de cinco años mencionado. Mucha de la variación ha sido debido a condiciones climatológicas (huracán Mitch en 1998 y una sequía severa en el 2000) que causaron una escasez de producción de frijol, consecuentemente un incremento en los precios regionales de frijoles rojos. Aún más, seguidamente a los desastres naturales, las importaciones y donaciones inundaron la región de frijoles, causando un exceso de oferta y un sub-secuente desplome de los precios (Figura 3).

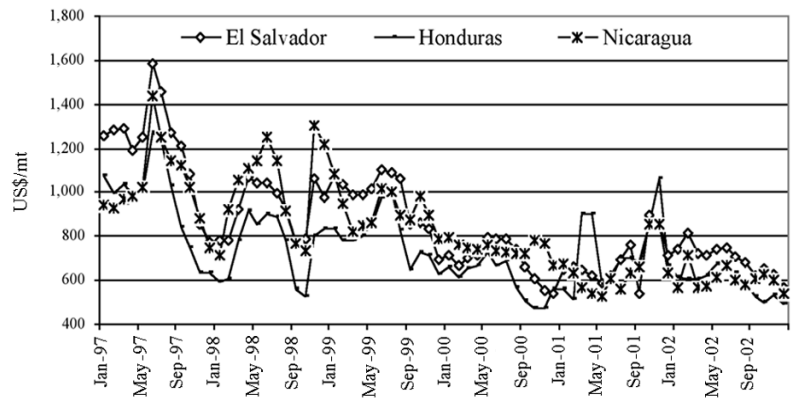

Figura 3. Precios nominales mayoristas de frijoles rojos. El Salvador, Honduras, y Nicaragua, 1997-2002.

Fuente: Datos de CORECA (2003).

Durante el periodo 1997-2002, el precio promedio en la temporada baja varió desde 826 US\$/t en Costa Rica a 626 US\$/t en Honduras; y el precio promedio en temporada alta varió de 981 US\$/t en El Salvador, a 824 US\$/t en Guatemala (Cuadro 5).

Cuadro 5. Precios de frijoles en temporadas bajas y altas. Centroamérica, 1997-2002.

\begin{tabular}{|c|c|c|c|c|c|c|}
\hline \multirow[t]{2}{*}{ País } & \multirow[t]{2}{*}{ Clase de frijol } & \multicolumn{2}{|c|}{ Temporada baja } & \multicolumn{2}{|c|}{ Temporada alta } & \multirow{2}{*}{$\begin{array}{c}\text { Porcentaje } \\
\text { de variación }{ }^{b}\end{array}$} \\
\hline & & Mes & Precio $^{a}$ & Mes & Precio $^{\mathbf{a}}$ & \\
\hline Costa Rica & Negro & Marzo & 826 & Julio & 890 & $7 \%$ \\
\hline Guatemala & Negro & Marzo & 686 & Julio & 824 & $17 \%$ \\
\hline El Salvador & Rojo & Diciembre & 711 & Junio & 981 & $28 \%$ \\
\hline Honduras & rojo & Setiembre & 626 & Julio & 861 & $27 \%$ \\
\hline Nicaragua & rojo & Diciembre & 767 & Junio & 953 & $19 \%$ \\
\hline
\end{tabular}

a Precio en US $\$ / t$

b Precio de temporada alta dividido por precio de temporada baja multiplicado por 100 .

Fuente: Precios de CORECA (2003). 
El patrón de estacionalidad de los precios observados durante este periodo, se debió al hecho que aproximadamente un $80 \%$ de la producción regional estuvo concentrada durante los meses de enero a agosto (CORECA 1999). La estacionalidad en la producción de frijol crea una serie de problemas, principalmente por la falta de condiciones de almacenamiento y los costos del mismo, acarreando que los productores tengan que vender toda su producción cuando existe cosecha. De esta manera, debido al exceso de oferta en la segunda mitad de cada año los precios declinan rápidamente. Como es de esperarse los precios del frijol rojo en El Salvador, Honduras y Nicaragua, empiezan a bajar después de julio y se incrementan después de setiembre. La variación de precios es especialmente observable en los frijoles rojos, posiblemente por la ausencia de buenos substitutos fuera de la región.

Contrariamente, en Costa Rica, donde la demanda es mayormente de frijoles negros, la existencia de buenos substitutos fuera de la región contribuye a que las importaciones representen aproximadamente $61 \%$ del total de frijoles consumidos en el país. Esto afecta que la variación de precios sea menos dramática durante el año. Sin embargo, en Guatemala, con las mismas característica de demanda que Costa Rica, pero con menor proporción de importaciones de frijoles, los precios disminuyen desde enero hasta junio y se incrementan desde julio hasta parte de agosto y nuevamente disminuyen desde setiembre hasta marzo (Cuadro 5).

\section{Precios internacionales}

Los precios internacionales mas relevantes para comparar la competitividad de los frijoles rojos y negros en Centro América son los precios de Argentina y de EE.UU. Mientras que para los frijoles negros existen adecuados substitutos fuera de la región, no existe otro país que actualmente produzca el tipo de frijol rojo apreciado en la región (CORECA 1999). Si bien la preferencia de los consumidores reduce la demanda de frijoles rojos importados, estos países todavía necesitan importar ya que la producción local no abastece la demanda.

Los países centroamericanos aplican diferentes aranceles a las importaciones de frijoles provenientes de países que se encuentran fuera de la región. Los aranceles varían desde un $20 \%$ a $30 \%$ en promedio. Para analizar el impacto de los aranceles en el precio de los frijoles, los precios de frijoles de EE.UU. y Argentina fueron comparados con los precios de los mayores productores de la región (Guatemala para los frijoles negros y Honduras y Nicaragua para frijoles rojos pequeños).
Para los frijoles negros en Guatemala, durante el periodo 1997-2002, la competitividad fue determinada comparando los precios promedios mensuales a nivel mayorista de los frijoles producidos localmente con los precios mayoristas de los EE.UU. (precios F.O.B), incluyendo los costos de transporte a Centro América, y excluyendo e incluyendo los aranceles de importaciones (Cuadro 6).

Cuadro 6. Costo estimado promedio de importación desde frijoles negros de Michigan, EE.UU. a Guatemala. Julio a septiembre, 2002.

\begin{tabular}{lc}
\hline \multicolumn{1}{c}{ Descripción de costos } & $\begin{array}{c}\text { Costo } \\
\text { (US\$/TM) }\end{array}$ \\
\hline Costos de frijoles negros (F.O.B) & 619 \\
Transporte, seguro y otros & 80 \\
Arancel & 186 \\
Costo Total & 885 \\
\hline
\end{tabular}

Fuente: Datos proporcionados por Letrimex (2003), Air Parcel Express (2003) y Eisengruber (2003).

Durante el periodo 1997-2002, la competitividad de los precios de frijoles en Guatemala varió, comparado con los precios de EE.UU. (Figura 4). Cuando se incluyen los aranceles, desde el cuarto trimestre de 1998 hasta el tercer trimestre del 2001, los precios de EE.UU. fueron similares a los de Guatemala. Sin embargo, después del tercer trimestre del 2001, los precios norteamericanos fueron significativamente más elevados (con aranceles). En contraste, cuando los aranceles no son incluidos, los precios de EE.UU. durante el periodo de seis años fueron significativamente menores que los precios de frijoles en Guatemala.

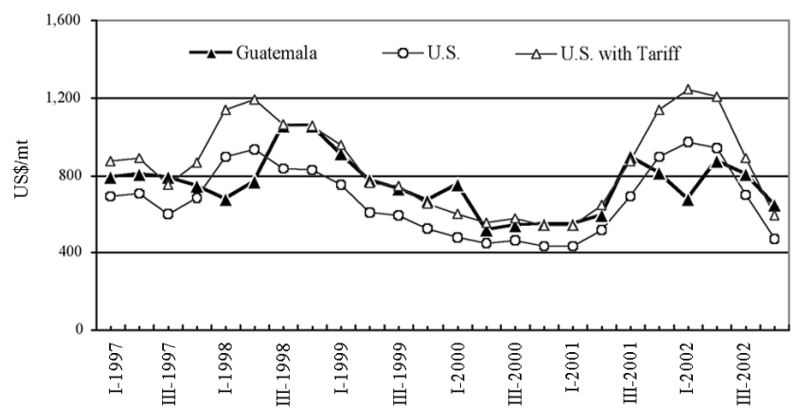

Figura 4. Precios promedios mayoristas de frijoles negros en Guatemala y EE.UU. con y sin aranceles. 1997-2002.

Fuente: Datos obtenidos de CORECA (2003) y USDA (2003). 
El precio más apropiado para determinar la competitividad internacional de los frijoles de Guatemala es el precio del frijol negro argentino. En el tercer trimestre del 2002, los importadores de frijoles podían comprar frijoles negros de Argentina a un precio substancialmente menor que los precios de Michigan (EE.UU.). El precio del frijol argentino (F.O.B) en el periodo mencionado fue de $393 \mathrm{US} \$ / \mathrm{t}$ (Cuadro 7), comparado con $800 \mathrm{US} \$ / \mathrm{t}$ por los frijoles de Guatemala ${ }^{3}$, y $619 \mathrm{US} \$ / \mathrm{t}$ por los frijoles de Michigan. En años recientes, los precios de Argentina fueron afectados principalmente por la devaluación del peso contra el dólar, por lo tanto la competitividad de los frijoles fue en parte debida a la volatilidad del peso más que a la productividad de los productores (Figura 5).

Cuadro 7. Costo estimado promedio de importación de frijoles negros desde Argentina a Guatemala. Julio a septiembre, 2002.

\begin{tabular}{lc}
\hline \multicolumn{1}{c}{ Descripción de costos } & $\begin{array}{c}\text { Costo } \\
\text { (US\$/TM) }\end{array}$ \\
\hline Costo de frijoles negros (F.O.B) & 393,33 \\
Transporte, seguro y otros & 124,25 \\
Arancel & 118,00 \\
Costo Total & 635,58 \\
\hline
\end{tabular}

Fuente: Datos proporcionados por Alcomex S.A, Argentina (Louzano 2003).

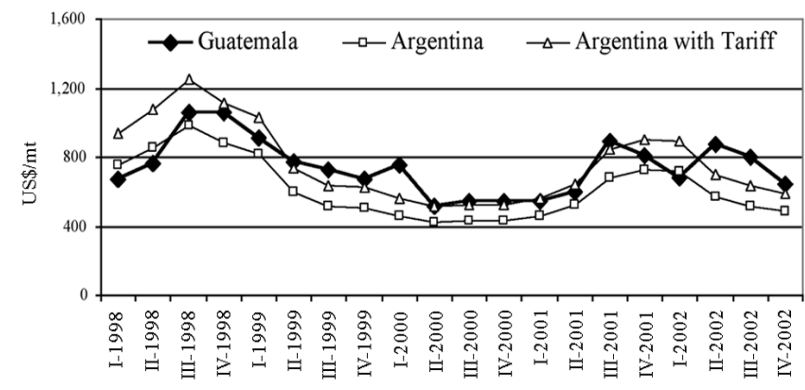

Figura 5. Precios promedios mayoristas de frijoles negros en Guatemala y Argentina con y sin aranceles. 1997-2002.

Fuente: Datos obtenidos de CORECA (2003) y la Secretaría de Agricultura de Argentina (2003).

\footnotetext{
3 Costo promedio de frijoles en el mercado mayorista de Guatemala desde julio hasta setiembre del 2002.
}

Para los frijoles rojos en Honduras y Nicaragua, la competitividad fue determinada comparando los precios mensuales de los frijoles rojos producidos localmente con los precios de frijoles rojos pequeños producidos en los EE.UU.4. Durante el periodo 1997-2002, los precios de frijoles fueron comparados excluyendo e incluyendo aranceles de importación. Los costos de transporte y los aranceles aduaneros fueron considerados iguales a los de los frijoles negros y los mismos fueron utilizados como referencia para los efectos del análisis del costo de exportación a Nicaragua y Honduras.

En el caso de Nicaragua, desde el segundo trimestre de 1997 hasta aproximadamente el tercer trimestre del 2000, los precios de frijoles rojos fueron más elevados que los precios de EE.UU. con aranceles incluidos. Sin embargo, después del 2000 los precios de Nicaragua decrecieron sustancialmente para ubicarse muy por debajo de los precios norteamericanos. En el caso de Honduras, los precios relativos fueron variables entre el segundo trimestre de 1998 hasta el primer trimestre del 2000. Si bien después del 2000 los precios de Honduras fueron menores comparados con los de EE.UU. con aranceles (Figura 6). Sin aranceles, los precios del frijol hondureño se ubicaron generalmente por debajo de los precios norteamericanos durante el periodo 1997 - 2002.

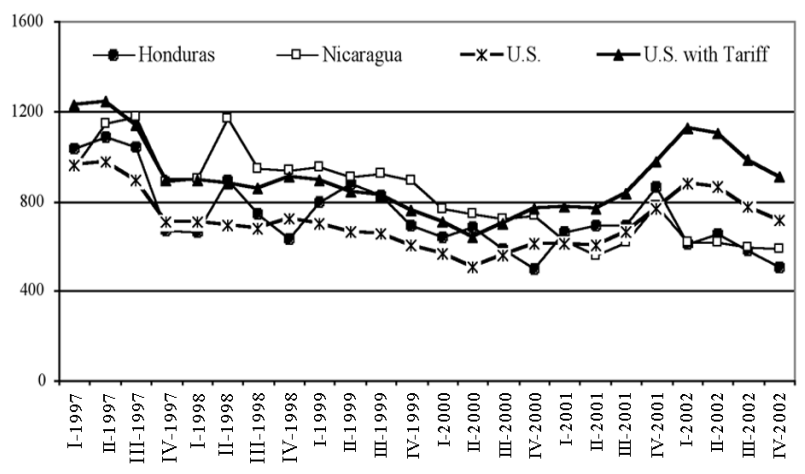

Figura 6. Precio promedio mayorista del frijol rojo pequeño en Honduras, Nicaragua y EE.UU. con y sin aranceles. 1997-2002.

Fuente: Datos de CORECA (2003) y USDA (2003).

$4 \mathrm{Si}$ bien los frijoles pequeños de EE.UU no son substitutos perfectos del frijol rojo pequeño de Centroamérica, éste es el que más se asemeja al frijol rojo centroamericano. 


\section{CONCLUSIONES}

La pregunta básica a la que se enfrentan los países centroamericanos es si serán o no competitivos en sus mercados, en el mercado regional y en el mercado internacional; no solo produciendo frijoles a precios competitivos, pero también con la calidad que es demandada por la industria y los consumidores. Los países centroamericanos han tratado de mantener su competitividad al imponer aranceles a las importaciones de frijoles no provenientes de la región, los mismos fueron de un rango del 20 a $30 \%$, pero estos aranceles han fallado en hacer a la región autosuficiente en la producción de frijoles y se han incrementado los precios que los consumidores finales pagaban innecesariamente.

Actualmente, los rendimientos de la producción de frijol siguen muy bajos, comparados con los rendimientos que EE.UU. y Argentina pueden obtener. Si bien muchos de los pequeños productores centroamericanos tienen muy poca posibilidad de expandir su producción de frijoles a un nivel comercial, los mismos continuarán produciéndolos principalmente por la importancia como un alimento de consumo básico y porque todavía es una fuente de ingresos. Para poder mantener cierta competitividad en un mercado cada vez más globalizado, es indispensable el incremento de los rendimientos, es por eso que los gobiernos centroamericanos y los donantes internacionales necesitan concentrar sus esfuerzos en la investigación para el desarrollo de variedades que tengan mayor rendimiento y que reduzcan el riesgo asociado a la producción de frijoles.

En toda la región existe un limitado porcentaje de adopción de variedades mejoradas de frijol. Si los productores no poseen un acceso mayor a las variedades mejoradas, la oferta de frijoles en la región continuará siendo insuficiente para competir con las importaciones. Los programas nacionales de distribución de semillas necesitan determinar rigurosamente las fortalezas y debilidades de sus esquemas de distribución de semillas para identificar las fallas y aprender de las lecciones de otros países en la región, por ejemplo el de la asociación entre el Programa de Investigación y Transferencia de Tecnología Agropecuaria en Frijol (PITTA- Frijol) de Costa Rica, grupos de productores y la Empresa Hortifruti Costa Rica S.A.

Durante los años 90, el mercado del frijol experimentó numerosos cambios, incluyendo la consolidación entre mayoristas y supermercados, las posibilidades de expansión de los mercados de exportación, la creciente demanda de productos con mayor valor agregado y la creciente preferencia por frijoles de mayor calidad, especialmente con respecto a la apariencia (color). Para asegurar que en el futuro las variedades puedan satisfacer las preferencias con respecto a calidad, existe la necesidad de establecer mecanismos que involucren a vendedores de frijoles (mayoristas, importadores, exportadores, supermercados). Los consejos de frijoles deben incluir a todos los miembros de la cadena desde la producción hasta la venta de frijoles. Los antiguos esquemas que promueven la eliminación de intermediarios no funcionan y no funcionarán si lo que se busca es acceder a información con respecto a los gustos de los consumidores.

Finalmente, los productores de frijoles, especialmente los pequeños y medianos, no se han beneficiado del crecimiento de la industria del valor agregado. Según los entrevistados el mayor obstáculo es la ausencia de volúmenes durante todo el año. Sin embargo, los productores también carecen de información acerca de los requerimientos de la industria y los estándares que imponen hoy en día. Los gobiernos, o entidades que ayudan a los productores, deberían asistir a los productores para que éstos puedan tener información actualizada relacionada a los estándares que la industria requiere. También monitorear que los productores puedan cumplir con los mismos y negociar con ellos y los miembros de la industria para asegurar una oferta constante.

\section{RECONOCIMIENTOS}

Esta publicación ha sido posible a través del apoyo del Programa Bean/Cowpea Collaborative Research Support Program (CRSP), auspiciado por la Agencia para el Desarrollo Internacional de los Estados Unidos de Norteamérica (United States Agency for International Development, USAID), Donación No. GDG-G-0002-00012-00. Las opiniones expresadas en este documento por los autores no reflejan necesariamente los puntos de vista de USAID.

Agradecemos al Dr. Juan Carlos Rosas de la Escuela Agrícola Panamericana, Zamorano, Honduras, por su colaboración en la edición de este documento.

\section{LITERATURA CITADA}

AIR PARCEL EXPRESS (APE). 2003. Instant Ocean Freight Calculator (on line). Consultado [Julio 30, 2003]. Disponible: http://www.shipping-worldwide.com/

BATRES-MARQUEZ, S. P.; JENSEN, H. H.; BRESTER, G. W. 2001. Salvadoran consumption of ethnic foods in the United States. Center for Agricultural and Rural Development Iowa State University Working Paper 01WP 289. Ames, Iowa. p. 16. 
CONSEJO REGIONAL CENTRO AMERICANO (CORECA). 1999. Comportamiento de los niveles arancelarios para los granos básicos durante el periodo 1995 - 1999. Reunión de Ministros del Consejo Agropecuario Centroamericano, Costa Rica. Disponible: http://www. coreca.org/documentos/02-99.pdf

CONSEJO REGIONAL CENTRO AMERICANO (CORECA). 2003. Precios de productos e insumos agropecuarios del CORECA (on line). Consultado [27 de agosto del 2003]. Disponible: http://www.coreca.org/sistema_informacion/precios/default.asp.

EISENGRUBER, M. 2003. 7211 E. Michigan Ave. Pigeon, Michigan 48755. Phone 517-453-4500. Contacto telefónico realizado el 30 julio 2003.

ESTRADA-VALLE, J. M. 2001. A sub-sector overview of the Guatemalan bean industry: Constraints and opportunities for vertical coordination and sustainable growth. Master of Science. Michigan State University.

HOLTZMAN, J. S. 1986. Rapid reconnaissance guidelines for agricultural marketing and food system research in developing countries. MSU International Development Papers Working paper No. 30: p 30. East Lansing, Michigan.
LETRIMEX. 2003. Online. Ocean Freigt Rates. Cosultado 24 Julio 2003. Disponible: http://www.letrimex.com/ oceanfreightrates.htm

LOUZANO, A. 2003. Correo electrónico: louzao@alcomex. com.ar. Alcomex SA, Buenos Aires, Argentina. Consultado el 24 julio 2003

MARTEL, P.; BERNSTEN, R.; WEBER, M. 2000. Food markets, policy, and technology: The case of honduran dry beans. MSU International Development Working Papers, no. 78: p1.

SECRETARIA DE AGRICULTURA, GANADERÍA, PESCA Y ALIMENTOS DE ARGENTINA (SAGP y A). 2003. Precios FOB oficiales de porotos negros. Promedio mensual 1997-2002. Consultado 24 julio 2003. Disponible: http://www.sagpya.mecon.gov.ar/00/index/agricultura/index_agricultura.htm

SECRETARIA DE INTEGRACIÓN DEL CONSEJO CENTRO AMERICANO (SIECA). 2003. Sistema de estadísticas de Centroamérica SEC. Consultado 27 agosto 2003. Disponible: http://www.sieca.org.gt/ SIECA.htm

UNITED STATE DEPARTMENT OF AGRICULTURE (USDA). 2003. Briefing room. Dry beans: market outlook. Consultado el 2 agosto 2003. Disponible: http://www.ers.usda.gov/briefing/drybeans/market.htm 